\title{
Getting to know the fetal genome non-invasively: now a reality
}

\author{
Maulik M Dhandha \\ School of Medicine, St. Louis University, 1402 S Grand Blvd, St. Louis, MO 63110, USA \\ $\triangle$ Correspondence: mdhandha@slu.edu
}

Prenatal screening tests of genetic disorders are routinely performed in modern day obstetrics to diagnose fetal genomic abnormalities including trisomies 13,18 and 21. These include amniocentesis and chorionic villi sampling, both of which are invasive testing processes and post a small but significant risk to the fetus (Evans and Wapner 2005). Other non-invasive methods such as pelvic ultrasound as well as maternal serum biomarkers measured at various phases of pregnancy are available to detect Trisomy 21 and some other congenital disorders (Malone et al., 2005). However, these tests are often designed for disease-specific diagnosis and not helpful in identifying defects at the whole genome level. As a result, other genetic disorders of the fetus are frequently missed by these traditional screening tests. In addition, these tests are not highly sensitive or specific to detect trisomies (Lo, 2012). An exciting recent study by HC Fan and her colleagues sought to tackle this problem and showed that it is possible to non-invasively determine the entire genome of the fetus prenatally (Fan et al., 2012).

Their work is based on the use of maternal plasma DNA, which has been intensively investigated during the past decades by numerous scientists. The phenomenon of circulating cell-free DNA in blood, which is termed as plasma DNA, was demonstrated by Mandel and Metais (1948). The exact source of plasma DNA in the blood remains unclear. Some scientists argue that it is released from necrotic and apoptotic cells (Gormally et al., 2007), while others believe that it is actively released from live cells (Anker et al., 1999; Stroun et al., 2001; Chen et al., 2005; Goebel et al., 2005; Rhodes et al., 2006). Regardless of the true source, plasma DNA seems to be very important for prenatal diagnosis and improving outcomes in maternal-fetal medicine.

Lo YMD and his colleagues were the first to report the presence of cell free fetal DNA in the maternal circulatory system during pregnancy (Lo et al., 1997). They demonstrated this by the presence of $\mathrm{Y}$ chromosomal sequences in the serum of pregnant females (Lo et al., 1997). Later, it was also shown that the amount of plasma DNA is 5-10 times higher in pregnant females when compared to normal healthy individuals (Hahn et al., 2009; Hung et al., 2009). This increases the yield of the DNA extraction process when applied to plasma from pregnant females. In addition, maternal plasma DNA is actually a mixture of maternal and fetal DNA. The fraction of fetal DNA in maternal plasma during pregnancy can range from a few percentages early in pregnancy to as high as $50 \%$ in later stages of gestation (Lo et al., 1998; Palomaki et al., 2012). This relatively high fetal fraction is the key to the newer non-invasive techniques for prenatal genome sequencing.

Recent advances in molecular biology such as digital PCR have allowed exploiting the plasma DNA for gaining more information of the human genome. The technique of digital PCR is an improvised version of the traditional PCR. It involves amplifying single target molecule by terminally diluting a solution of DNA, first performed by Sykes et al (1992) and later termed "digital PCR" by Vogelstein and Kinzler (1999). In simple terms, first a reaction mixture similar to the traditional PCR is prepared. This is then added to small wells/compartments in such a way that each well/compartment has less than one copy (approximately half a copy) of the template that is being sequenced. The positive signals are then counted and converted to a linear digital signal (Fig. 1). This method allows high precision and accuracy at determining the genomic sequence primarily.

This technique now even allows identifying hemoglobinopathies (Lun et al., 2008) and hemophilia (Lo et al., 1999) by calculating the relative dosage of mutant and normal haplotypes in maternal serum samples. By applying the relative dosage concept genome wide, Lo and colleagues have shown that genome-wide genetic and mutational profiles of the fetus can be determined (Lo et al., 2010). Using the in formation from both the paternal and maternal genetic maps, 
A
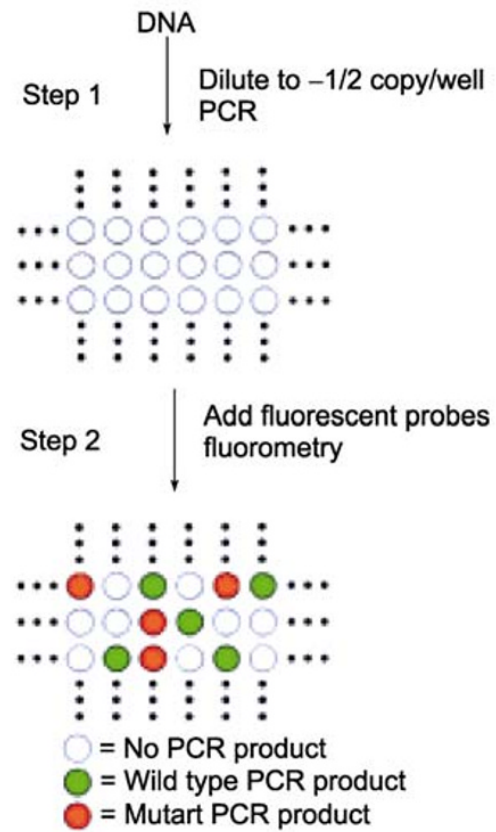

Figure 1. Given above is an illustration of how digital-PCR works (Vogelstein and Kinzler 1999, Copyright PNAS 1999).

this technique can be used to determine the entire fetal genome. This would make it possible to predict disease profiles and their risks factors before birth.

However, the unique aspect of the study by Fan and colleagues is that, they demonstrated for the first time that acquiring the entire fetal genomic information is possible without having the paternal genetic map (Fan et al., 2012). This is extremely important in modern day world, where single parenthood and unknown paternity are common.

In this study, Fan and colleagues used molecular counting of haplotypes using maternal plasma DNA to obtain the whole genome map of the fetus non-invasively. The fetal genome is a combination of four parental chromosomes, or haplotypes, that result from the process of random assortment as well as homologous recombination during the process of meiosis. Of the four fetal haplotypes, three haplotypes per genomic region are found in maternal serum during pregnancy (Fan et al., 2012). These include the maternal haplotypes that are transmitted to the fetus, the maternal haplotypes that are not transmitted to the fetus and the paternal haplotype that is transmitted (Fan et al., 2012). They first identified all the maternal haplotypes by obtaining maternal lymphocyte cells from pregnant females and isolating individual chromosomes from them (Fan et al., 2012). Once the maternal genome was known, the inheritance of the haplotypes in the fetus was then determined. This was accomplished by sequencing the maternal plasma DNA and determining the relative count of each allele at heterozygous loci (Fan et al., 2012).
In the same study, the paternal haplotypes were deciphered by detection of paternal-specific (aka non-maternal) alleles in the serum and imputation at the linked positions. Imputation literally means substituting some value for unknown or missing information. Genetic imputation, thus involves substituting genetic data for unknown or missing one. In their experiments, HC Fan and colleagues used haplotypes of normal population documented using the 1000 genome project for the imputation at the linked sites (Fan et al., 2012). They were able to detect $66 \%-67 \%$ of all the paternal (aka non-maternal) alleles in the plasma DNA from the pregnant females. These markers were then used for imputation of about $70 \%$ of paternal haplotypes with about $94 \%-97 \%$ accuracy (Fan et al., 2012). At least in theory, the remaining haplotypes that could not be imputed could be determined using deeper sequencing and applying the molecular counting principle directly to the individual alleles at every genomic position. Thus, the entire genomes of the fetus could be determined by using maternal plasma DNA and few lymphocytes from the blood of pregnant females. In their experiments, the group studied two pregnant patients. They were able to detect DiGeorge syndrome in the fetus of one of the pregnant patients by finding a $2.85-\mathrm{Mb}$ deletion of chromosome 22q11 (Fan et al., 2012).

In lines with the same goal, HC Fan and colleagues also showed an alternative approach to determine the whole genome of the fetus. This method involved capturing exome sequences from the plasma DNA and enriching them. Exomes are the true coding sequences interrupted by introns, the intervening sequences. Exomes are a couple of orders of magnitude smaller than the whole genome (Fan et al., 2012). Thus, they allow deeper sequencing at individual heterozygous loci (Fan et al., 2012). This in turn provides increased sensitivity and specificity in detection of paternal haplotypes or de novo mutations in the fetal genotype (Fan et al., 2012). Once the exomes were enriched; the same principle of DNA molecular counting was implemented by targeted deep sequencing to determine the fetal genome (Fan et al., 2012).

These recent advancements show that non-invasive fetal genetic testing may potentially play important roles in future obstetric practices. It would enable detection of medical conditions such as phenylketonuria, galactosemia, maple syrup urine disease and Severe Combined Immune Deficiency (Lo, 2012). These advancements, however, raise several ethical, social and economic questions. First of all, administering these tests on a large scale is not economically viable. Therefore it would be hard to provide such testing to all pregnant patients until they are made clinically cost-effective. In addition, knowing the diagnosis prenatally may put parents of the fetus in ethical dilemmas about whether or not to further the pregnancy. Not only that, but knowing the genetic makeup prior to birth might predispose the child to socioeconomic biases. One such example would be health care insurances exploiting this data to exclude clientele based on 
known genetic risk factors. But despite all this, non-invasive prenatal detection of genetic disorders has opened several doors into our understanding of disease inheritance and potential therapeutic interventions.

\section{ACKNOWLEDGEMENTS}

The author would like to thank Dr. Yan Yin for reviewing the article before submission.

\section{REFERENCES}

Anker, P., Mulcahy, H., Chen, X.Q., and Stroun, M. (1999). Detection of circulating tumour DNA in the blood (plasma/serum) of cancer patients. Cancer Metastasis Rev 18, 65-73.

Chen, Z., Fadiel, A., Naftolin, F., Eichenbaum, K.D., and Xia, Y. (2005). Circulation DNA: biological implications for cancer metastasis and immunology. Med Hypotheses 65, 956-961.

Evans, M.I., and Wapner, R.J. (2005). Invasive prenatal diagnostic procedures. Semin Pernatol 29, 215-218.

Fan, H.C., Gu, W., Wanf, J., Blumenfeld, Y.J., El-Sayed, Y.Y., and Quake, S.R. (2012). Non-invasive prenatal measurement of the fetal genome. Nature 487, 320-324.

Goebel, G., Zitt, M., Zitt, M., and Müller, H.M. (2005). Circulating nucleic acids in plasma or serum (CNAPS) as prognostic and predictive markers in patients with solid neoplasias. Dis Markers 21, 105-120.

Gormally, E., Caboux, E., Vineis, P., and Hainaut, P. (2007). Circulating free DNA in plasma or serum as biomarker of carcinogenesis: practical aspects and biological significance. Mutat Res 635 , 105-117.

Hahn, S., Jackson, L.G., Kolla, V., Mahyuddin, A.P., and Choolani, M. (2009). Noninvasive prenatal diagnosis of fetal aneuploidies and Mendelian disorders: new innovative strategies. Expert Rev Mol Diagn 9, 613-621.

Hung, E.C., Chiu, R.W., and Lo, Y.M. (2009). Detection of circulating fetal nucleic acids: a review of methods and applications. J Clin Pathol 62, 308-313.

Lo, Y.M., Tein, M.S., Lau, T.K., Haines, C.J., Leung, T.N., Poon, P.M., Wainscoat, J.S., Johnson, P.J., Chang, A.M., and Hjelm, N.M. (1998). Quantitative analysis of fetal DNA in maternal plasma and serum: implications for noninvasive prenatal diagnosis. Am J Hum
Genet 62, 768-775

Lo, Y.M., Chan, K.C., Sun, H., Chen, E.Z., Jiang, P., Lun, F.M., Zheng, Y.W., Leung, T.Y., Lau, T.K., Cantor, C.R., et al. (2010). Maternal plasma DNA sequencing reveals the genome-wide genetic and mutational profile of the fetus. Sci Transl Med 2, 1 ra91.

Lo, Y.M., Corbetta, N., Chamberlain, P.F., Rai, V., Sargent, I.L., Redman, C.W.G., and Wainscoat, J.S. (1997). Presence of fetal DNA in maternal plasma and serum. Lancet 350, 485-487.

Lo, Y.M., Zhang, J., Leung, T.N., Lau, T.K., Chang, A.M., and Hjelm, N.M. (1999). Rapid clearance of fetal DNA from maternal plasma. Am J Hum Genet 64, 218-224.

Lo, Y.M. (2012). Non-invasive prenatal diagnosis by massively parallel sequencing of maternal plasma DNA. Open Biol 2. (In Press)

Lun, F.M.F., Chiu, R.W.K., Chan, K.C.A., Leung, T.Y., Lau, T.K., and Lo, Y.M.D. (2008). Microfluidics digital PCR reveals a higher than expected fraction of fetal DNA in maternal plasma. Clin Chem 54, 1664-1672.

Malone, F.D., Canick, J.A., Ball, R.H., Nyberg, D.A., Comstock, C.H., Bukowski, R., Berkowitz, R.L., Gross, S.J., Dugoff, L., Craigo, S.D., et al. (2005) First-trimester or second trimester screening, or both, for Down's syndrome. N Engl J Med 353, 2001-2011.

Mandel, P., and Metais, P. (1948). Les acides nucleiques du plasm sanguine chez l'homme. C.R. Acad Sci Paris 142, 241-243.

Palomaki, G.E., Deciu, C., Kloza, E.M., Lambert-Messerlian, G.M., Haddow, J.E., Neveux, L.M., Ehrich, M., van den Boom, D., Bombard, A.T., Grody, W.W., et al. (2012). DNA sequencing of maternal plasma reliably identifies trisomy 18 and trisomy 13 as well as Down syndrome: an international collaborative study. Gene Med 14, 296-305

Rhodes, A., Wort, S.J., Thomas, H., Collinson, P., and Bennett, E.D. (2006). Plasma DNA concentration as a predictor of mortality and sepsis in critically ill patients. Crit Care 10, R60.

Stroun, M., Lyautey, J., Lederrey, C., Olson-Sand, A., and Anker, P. (2001). About the possible origin and mechanism of circulating DNA apoptosis and active DNA release. Clin Chim Acta 313, 139-142.

Sykes, P.J., Neoh, S.H., Brisco, M.J., Hughes, E., Condon, J., and Morley, A.A. (1992). Quantitation of targets for PCR by use of limiting dilution. Biotechniques 13, 444-449.

Vogelstein, B., and Kinzler K.W. (1999). Digital PCR. Proc Natl Acad Sci U S A 96, 9236-9241. 